\title{
VARIAÇÃO MORFOMÉTRICA DE ARCÍDEOS (MOLLUSCA: BIVALVIA) DA COSTA NORTE-NORDESTE DO BRASIL
}

\author{
ROCHA, V. P. ${ }^{1 *} \&$ MATTHEWS-CASCON, H. ${ }^{2}$ \\ 1. Doutora em Ciências Marinhas Tropicais, Instituto de Ciências do Mar (LABOMAR/UFC), Labo- \\ ratório de Invertebrados Marinhos do Ceará (LIMCE) \\ 2. Professora Doutora da Universidade Federal do Ceará (UFC), Laboratório de Invertebrados Marin- \\ hos do Ceará (LIMCE)" \\ *Corresponding author: rocha.vp16@gmail.com
}

\begin{abstract}
Rocha, V.P. \& Matthews-cascon, H. (2014). Variação morfométrica de Arcídeos (Mollusca: Bivalvia) da costa nortenordeste do Brasil. braz. J. Aquat. Sci. Technol. 19(1). elSSN 1983-9057. DOI: 10.14210/bjast.v19n1. Known as "ark-shells" and "blood cookles", species of family Arcidade are sessile and it's very common in tropical seas. The study aiming check the shells morphological metric elements that would help in arcids differentiation (Mollusca: Bivalvia: Arcidae), as well as examine the conchological variations of specimens from North and Northeastern regions of Brazil. The samples available were from malacological collection "Prof. Henry Ramos Matthews" (CMPHRM), of Universidade Federal do Ceará (UFC), wich only the right valve was used. After shells identification, eight measures were taken: hinge length, shell length, position of umbo, umbo height, anterior shell height, middle shell height, posterior shell height and posterior shell length. Those measures are previously known as 'landmarks' of the family. The data were analyzed by statistical packages and shell's mean sizes were consistent with measures presented in the literature. In general, the measures were significant in the differentiation of species. Different specimens with geographical proximity it often have morphological similarity.
\end{abstract}

Key words: Morphological patterns, Similarity, Shells, Arcidae.

\section{INTRODUÇÃO}

As espécies da família Arcidade tem como características concha inequilateral, de formato ovalado a subtrapezoidal, com escultura radial ou decussata, porcelanosa e com numerosos dentes taxodontes localizados em uma charneira reta, além de perióstraco espesso e ocasionalmente piloso (Rios, 1994, 2009; Amaral et al., 2006). São também conhecidas como "ark-shells" e "blood cookles".

Bastante comum em mares tropicais, para o Brasil registram-se 21 espécies para a família, classificadas nos gêneros Acar, Arca, Anadara, Aspearca, Barbatia, Bathyarca, Bentharca e Paranadara (Diaz \& Puyana, 1994; Simone \& Chichvarkhin, 2004; Passos \& Birman, 2009; Rios, 1994, 2009; Simone, 2009; Francisco et al., 2012). Distribuídas por toda a costa brasileira, algumas espécies não ocorrem na região Sul do país (Rios, 2009).

Importantes ecológica e economicamente são consideradas fonte de proteína para o homem, sendo conhecidas popularmente como 'mariscos' juntamente a outras espécies de bivalves. Para o Brasil registrase Anadara chemnitzi (Philippi, 1851) como espécie comestível na região Nordeste (Rios, 2009). Em alguns países há o reconhecimento do potencial de espécies desta família para a aquicultura e setor pesqueiro (Broom, 1983; Peharda et al., 2006; Silina, 2006). No ambiente estão presentes nas cadeias alimentares, bem como na formação do substrato marinho.
Os arcídeos, de um modo geral, são animais sésseis e possuem concha bastante sujeita a variações ecofenotípicas, podendo apresentar diversidade de características para melhor adaptação a dinâmica ambiental em que se encontram (Simone \& Chichvarkhin, 2004). Essas alterações podem ser na taxa de crescimento, espessura e ornamentação das conchas, dentre outras.

Para estudos de variabilidade geográfica, ecomorfológicos e paleontológicos, bem como auxílio na resolução de problemas taxonômicos de identificação de espécies similares, os métodos de morfometria geométrica apresentam-se como ferramentas eficientes em análises com bivalves (Fuiman et al., 1999; Marko \& Jackson, 2001; Roopnarine et al., 2008; La Perna \& D'Abramo, 2009; Francisco et al., 2011). Essas análises levam em consideração 'marcos anatômicos' (landmarks), que, matematicamente, são pontos de correspondência combinados dentro e entre populações (Monteiro \& Reis, 1999; Rohlf, 2000; Zelditch et al., 2004).

Diferente da morfometria tradicional, cujas medidas eram escolhidas aleatoriamente, a morfometria geométrica baseadas em marcos anatômicos nos dá a possibilidade de observarmos as variáveis morfológicas da concha especificamente para determinado grupo. Estes dados morfológicos nos mostram possíveis relações de similaridade entre espécies e podem ser utilizados para indicar distinção de espécies próximas, quer sejam do Recente ou fósseis (ver 
Fuiman et al., 1999; Marko \& Jackson, 2001; Francisco et al., 2011).

Moluscos bivalves necessitam de mais estudos taxonômicos para serem estabelecidos em um sistema natural, o que não acontece em geral devido a uma grande variedade morfológica e ecológica, (Purchon \& Brown, 1969; Nevesskaja, 2009; Bieler et al., 2013). Oliver \& Holmes (2006) afirmam, após estudo sistemático da ordem Arcoidae, a necessidade de mais dados morfológicos para resolver as relações da família Arcidade, principalmente quando comparadas a família Noetiidae. Estudos conquiológicos, principalmente quando se tratam de novas espécies, são difíceis devido à falta de consenso na definição de gêneros e a disposição destes dentro da família Arcidae (Oliver \& Holmes, 2006; Francisco et al., 2012).

Desta forma, diante da importância de ampliação de dados taxonômicos, o presente estudo tem como objetivo verificar variáveis morfológicas da concha que possam auxiliar na diferenciação de Arcidae (Mollusca: Bivalvia), bem como examinar as variações conquiológicas entre espécimes dos estados da região Norte e Nordeste do Brasil.

\section{MATERIAIS E METODOS}

Os espécimes analisados encontram-se depositados na Coleção Malacológica "Prof. Henry Ramos Matthews - Séries A e B" da Universidade Federal do Ceará (CMPHRM - Série A e B), e foram coletados na região Norte (estados do Amapá e Pará) e Nordeste (estados do Maranhão, Ceará, Rio Grande do Norte, Paraíba, Pernambuco e Bahia) do Brasil - em expedições e pesquisas científicas desde os anos de 1970 até a atualidade. As conchas são conservadas a seco e espécimes com partes moles em álcool etílico $70 \%$.

O material malacológico foi identificado de acordo com a literatura existente (Warmke \& Abbott, 1962; Abbott, 1965; Abbott \& Dance, 1983; Diaz \& Puyana, 1994; Rios 1994, 2009; Amaral et al., 2006). Algumas espécies, embora com registro na literatura para os estados das regiões Norte e Nordeste, não foram aqui estudadas por ausência de material na coleção malacológica da UFC ou número insuficiente de valvas (inferior a cinco). Conchas de espécimes juvenis não foram consideradas por muitas vezes mostrarem ornamentações diferentes do adulto, apresentando características mais genéricas do que específicas da espécie.

A classificação sistemática utilizada segue a proposta de Rios (2009). A espécie L Lnarca ovalis foi analisada dentro de Anadara, para observação da relação desta com outras espécies do gênero. Anteriormente, a espécie pertencia ao gênero Anadara sendo recentemente reclassificada no gênero Lunarca por Huber (2010) (Tabela I).

Para as análises morfométricas foram consideradas apenas as valvas direitas das conchas para se ter uma padronização. Foram medidas oito variáveis morfológicas, com auxílio de paquímetro $(0,1 \mathrm{~mm}$ de acurácia), baseadas no trabalho de Marko \& Jackson (2001): (1) comprimento da charneira; (2) comprimento da concha; (3) posição do umbo (prodisoconcha); (4) altura do umbo (prodisoconcha); (5) altura da porção

Tabela I - Classificação das espécies de Arcidae observadas nesse estudo, segundo sistemática proposta por Rios (2009), com atualização da espécie Lunarcar ovalis feita por Huber (2010).

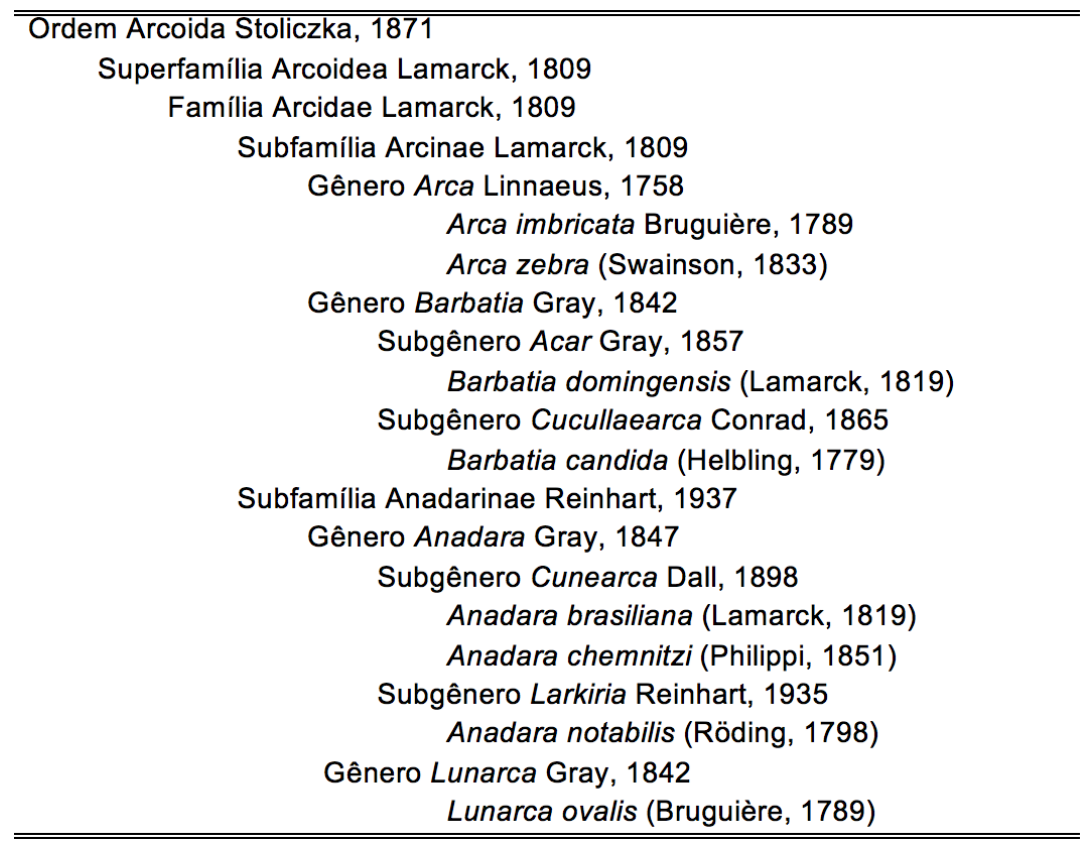




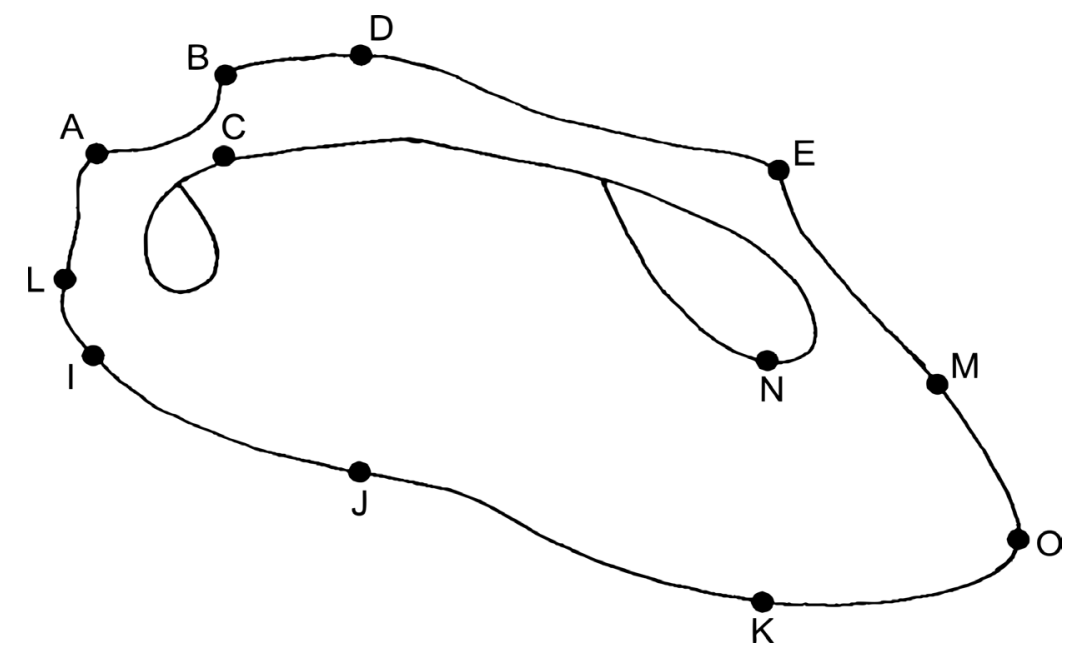

Figura 1- Características morfológicas utilizadas na diferenciação de conchas de bivalves arcídeos. As letras A, B, C, D, E, I, J, K, L, M, $\mathrm{N}$ e $\mathrm{O}$ indicam os marcos anatômicos. Variáveis morfológicas medidas neste estudo: comprimento da charneira (AE); comprimento da concha (LM); posição do umbo (prodisoconcha) (AC); altura do umbo (prodisoconcha) (CB); altura da porção anterior da concha (Al); altura média da concha (DJ); altura da porção posterior da concha (EK); comprimento da porção posterior da concha (NO) FONTE: Adaptado de Marko \& Jackson, 2001.

anterior da concha; (6) altura média da concha; (7) altura da porção posterior da concha; (8) comprimento da porção posterior da concha (NO) (Figura 1).

As variâncias foram analisadas através do programa estatístico GraphPad InStat $3 \circledR$. Para os resultados paramétricos, foi realizada uma análise de variância (ANOVA) e pós-teste de Turkey, enquanto que para resultados não paramétricos foi realizado teste de o Kruskal-Wallis e pós-teste de Dunn.

Também foram construídos dendrogramas de similaridades, por distância Euclidiana das médias das variáveis morfológicas, para espécies em estados brasileiros diferentes. Apenas uma valva de Arca imbricata do estado de Pernambuco foi analisada, sendo, desta forma, desconsiderada para a análise de similaridade.

\section{RESULTADOS}

Foram analisadas 414 valvas de oito espécies de arcídeos presentes na costa brasileira neste trabaIho. As espécies estudadas foram: Anadara chemnitzi (Philippi, 1851), dos estados do Pará e Ceará (15 valvas); Anadara brasiliana (Lamarck, 1719) do Ceará, Rio Grande do Norte e Paraíba (52 valvas); Anadara notabilis (Röding, 1798) do Pará, Ceará e Rio Grande do Norte (56 valvas); Arca imbricata (Bruguière, 1789) do Ceará, Rio Grande do Norte e Pernambuco (52 valvas); Arca zebra (Swainson, 1833) do Amapá, Pará, Maranhão e Ceará (92 valvas); Barbatia candida (Helbling, 1779) do Amapá (sete valvas); Barbatia domingensis (Lamarck, 1819) do Amapá, Pará, Ceará e Rio Grande do Norte (56 valvas); e Lunarca ovalis (Bruguière, 1789) do Pará, Ceará, Rio Grande do Norte e Paraíba (84 valvas) (Figura 2).
As medidas morfométricas variaram entre paramétricas e não paramétricas de acordo com os grupos analisados (Tabelas II a V). Valores de $p>0,05$ foram considerados não significantes, enquanto valores significantes tiveram sua relevância na separação das espécies dividida em: alta $(p<0,001)$, moderada $(p<0,01)$ ou baixa $(p<0,05)$. De forma geral, as medidas apresentaram-se significantes na diferenciação das espécies dentro dos gêneros e entre os subgêneros e o gênero Lunarca.

$\mathrm{Na}$ análise do gênero Anadara (incluindo o gênero Lunarca) com exceção do comprimento da concha, todas as outras variáveis morfológicas apresentaram-se não significantes para diferenciação de algumas espécies entre si (Tabela II). Nos gêneros Arca e Barbatia todas as medidas são significantes na distinção das espécies, onde a medida da altura do umbo (prodisoconcha), embora significante, teria menor relevância (Tabelas III e IV).

A análise de variância mostrou que as variáveis morfológicas altura do umbo (prodisoconcha), altura média, altura da porção posterior e o comprimento da concha foram significantes na distinção dos subgêneros Larkiria e Cunearca e destes com o gênero Lunarca. As outras medidas não foram significantes em todas as análises para distinção de Cunearca (Tabela V).

Observou-se a formação de três grupos, de acordo com o dendrograma de similaridade baseado nas variáveis morfológicas das conchas: o primeiro grupo une Anadara chemnitzi e Lunarca ovalis; o segundo une Arca zebra, Barbatia candida e Barbatia domingensis; e o terceiro grupo une Anadara brasiliana, Anadara notabilis e Arca imbricata (Figura 3). 


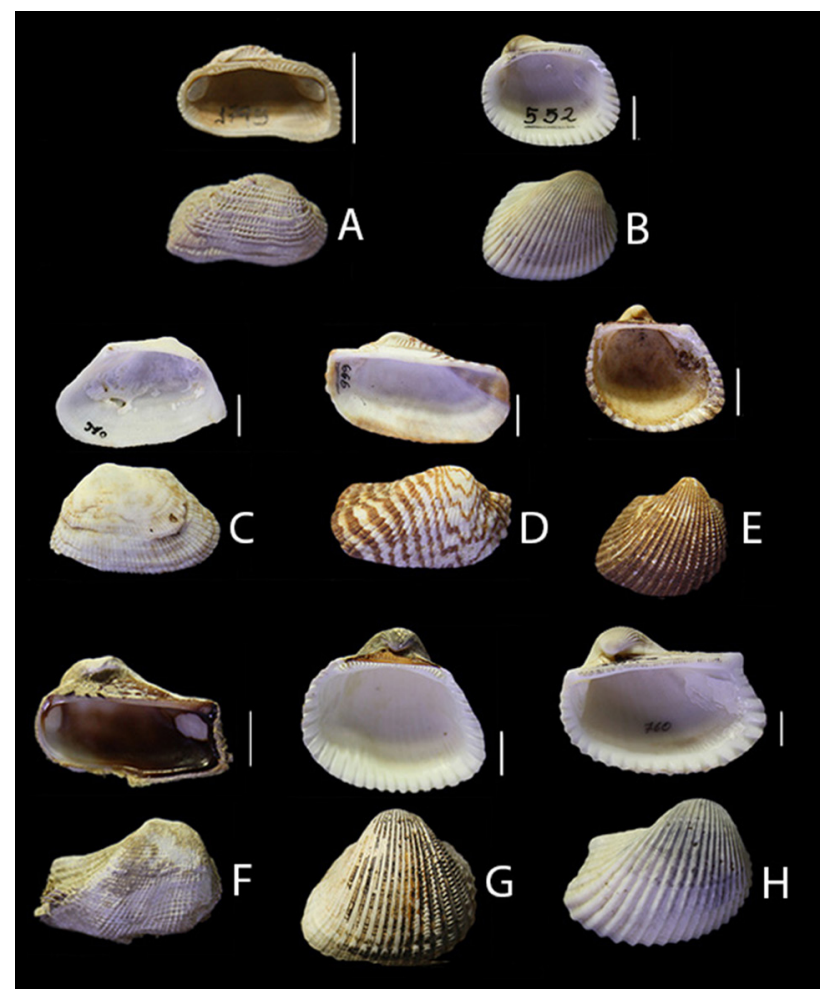

Figura 2- Visão interna (superior) e externa (inferior) das conchas das espécies de arcídeos analisados neste estudo. Escala: $1 \mathrm{~cm}$. Legenda: A- Barbatia domingensis; B- Lunarca ovalis; C- Barbatia candida; D- Arca zebra; E- Anadara chemnitzi; F- Arca imbricata; G- Anadara brasiliana; H- Anadara notabilis.

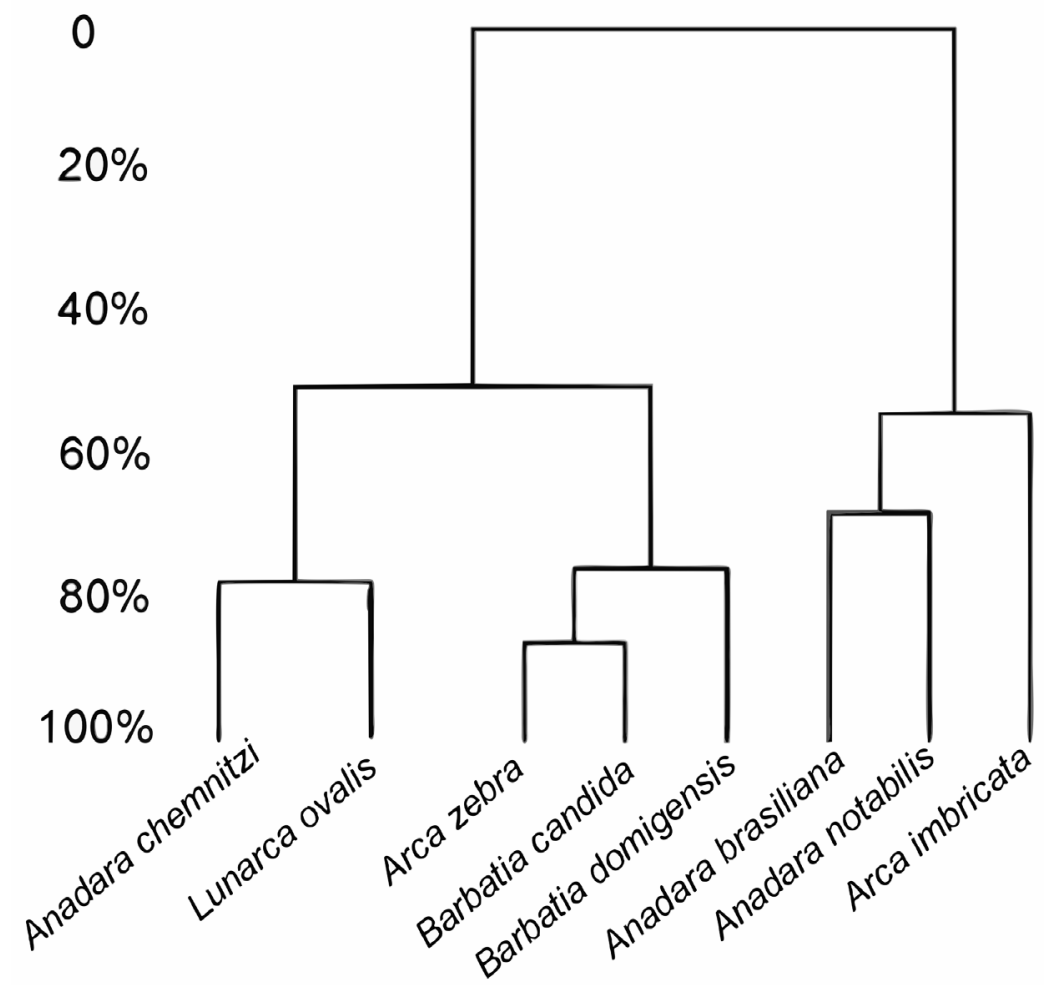

Figura 3 - Dendrograma de similaridade por distância Euclidiana das oito variáveis morfológicas medidas em arcídeos da costa NorteNordeste brasileira, mostrando uma possível relação entre as espécies. 
Tabela II - Análises de variância entre as espécies do Gênero Anadara, incluindo Lunarca ovalis, de acordo com as medidas morfométricas da concha consideradas no estudo. Legendda: NS- não significante; Asteriscos- significantes, onde: $\left({ }^{*}\right)$ baixa relevância, $\left(^{* *}\right)$ moderada relevância e $\left.{ }^{* * *}\right)$ alta relevância.

\begin{tabular}{|c|c|c|c|}
\hline $\begin{array}{l}\text { Medida } \\
\text { (variável) }\end{array}$ & $\begin{array}{l}\text { Teste } \\
\text { Paramétrico }\end{array}$ & Comparação & $P$ value \\
\hline & & Anadara chemnitzi x Anadara brasiliana & **** $p<0.001$ \\
\hline & & Anadara chemnitzi $x$ Lunarca ovalis & NS $p>0.05$ \\
\hline AC & Paramétrico & Anadara chemnitzi $x$ Anadara notabilis & $* * * \quad p<0.001$ \\
\hline (Posição & & Anadara brasiliana $x$ Lunarca ovalis & $* * * \quad p<0.001$ \\
\hline \multirow[t]{4}{*}{ do umbo) } & & Anadara brasiliana $x$ Anadara notabilis & $* * * \quad p<0.001$ \\
\hline & & Lunarca ovalis $x$ Anadara notabilis & $* * * \quad p<0.001$ \\
\hline & & Anadara chemnitzi x Anadara brasiliana & *** $\quad p<0.001$ \\
\hline & & Anadara chemnitzi x Anadara ovalis & $p<0.01$ \\
\hline $\mathrm{AE}$ & Não & Anadara chemnitzi x Anadara notabilis & $* * * \quad p<0.001$ \\
\hline (Comp. & Paramétrico & Anadara braziliana $x$ Lunarca ovalis & NS $p>0.05$ \\
\hline \multirow[t]{3}{*}{ charneira) } & & Anadara braziliana $x$ Anadara notabilis & $* * * \quad p<0.001$ \\
\hline & & Lunarca ovalis $x$ Anadara notabilis & $* * * \quad p<0.001$ \\
\hline & & Anadara chemnitzi x Anadara brasiliana & $* * * \quad p<0.001$ \\
\hline Al & & Anadara chemnitzi $x$ Lunarca ovalis & NS $p>0.05$ \\
\hline (Altura da & & Anadara chemnitzi x Anadara notabilis & $* * * \quad p<0.001$ \\
\hline porção & Paramétrico & Anadara brasiliana $x$ Lunarca ovalis & $* * * \quad p<0.001$ \\
\hline anterior & & Anadara brasiliana $x$ Anadara notabilis & NS $p>0.05$ \\
\hline \multirow[t]{3}{*}{ da concha) } & & Lunarca ovalis $x$ Anadara notabilis & $* * * \quad p<0.001$ \\
\hline & & Anadara chemnitzi x Anadara brasiliana & NS $p>0.05$ \\
\hline & & Anadara chemnitzi x Anadara ovalis & $* p<0.05$ \\
\hline CB & Não & Anadara chemnitzi $x$ Anadara notabilis & ** $p<0.01$ \\
\hline (Altura do & Paramétrico & Anadara brasiliana $x$ Lunarca ovalis & $* * * \quad p<0.001$ \\
\hline \multirow[t]{4}{*}{ umbo) } & & Anadara brasiliana $x$ Anadara notabilis & $* * * \quad p<0.001$ \\
\hline & & Lunarca ovalis $x$ Anadara notabilis & $* * * \quad p<0.001$ \\
\hline & & Anadara chemnitzi x Anadara brasiliana & *** $\quad p<0.001$ \\
\hline & & Anadara chemnitzi x Lunarca ovalis & NS $p>0.05$ \\
\hline DJ & Não & Anadara chemnitzi x Anadara notabilis & $* * * \quad p<0.001$ \\
\hline (Altura & Paramétrico & Anadara brasiliana $\times$ Lunarca ovalis & $* * * \quad p<0.001$ \\
\hline média & & Anadara brasiliana $x$ Anadara notabilis & NS $p>0.05$ \\
\hline da concha) & & Lunarca ovalis $x$ Anadara notabilis & $* * * \quad p<0.001$ \\
\hline
\end{tabular}


Braz. J. Aquat. Sci. Technol., 2015, 19(1).

Tabela III - Análises de variância entre as espécies do Gênero Arca de acordo com as medidas morfométricas da concha consideradas no estudo. Legendda: NS- não significante; Asteriscos- significantes, onde: $\left(^{*}\right)$ baixa relevância, $\left({ }^{* *}\right)$ moderada relevância e $\left(^{* * *}\right)$ alta relevância.

\begin{tabular}{|c|c|c|c|}
\hline Medida (variável) & $\begin{array}{l}\text { Teste } \\
\text { Paramétrico }\end{array}$ & Comparação & $P$ value \\
\hline $\begin{array}{c}\mathbf{A C} \\
\text { (Posição do umbo) }\end{array}$ & Não Paramétrico & Arca imbricata x Arca zebra & $* * * \quad p<0.001$ \\
\hline $\begin{array}{c}\mathrm{AE} \\
\text { (Comp. charneira) }\end{array}$ & Não Paramétrico & Arca imbricata x Arca zebra & ${ }^{* * *} p<0.001$ \\
\hline $\begin{array}{c}\text { Al } \\
\text { (Altura da porção } \\
\text { anterior da concha) }\end{array}$ & Não Paramétrico & Arca imbricata x Arca zebra & *** $p<0.001$ \\
\hline $\begin{array}{c}\mathbf{C B} \\
\text { (Altura do umbo) }\end{array}$ & Não Paramétrico & Arca imbricata x Arca zebra & $* * * \quad p<0.001$ \\
\hline $\begin{array}{c}\text { DJ } \\
\text { (Altura média da } \\
\text { concha) }\end{array}$ & Não Paramétrico & Arca imbricata x Arca zebra & *** $p<0.001$ \\
\hline $\begin{array}{c}\text { EK } \\
\text { (Altura da porção } \\
\text { posterior da concha) }\end{array}$ & Não Paramétrico & Arca imbricata x Arca zebra & $* * * \quad p<0.001$ \\
\hline $\begin{array}{c}\text { LM } \\
\text { (Comp. da concha) }\end{array}$ & Não Paramétrico & Arca imbricata x Arca zebra & $* * * \quad p<0.001$ \\
\hline $\begin{array}{c}\text { NO } \\
\text { (Comp. da porção } \\
\text { posterior da concha) }\end{array}$ & Não Paramétrico & Arca imbricata x Arca zebra & $* * * \quad p<0.001$ \\
\hline
\end{tabular}

Tabela IV . Análises de variância entre as espécies do Gênero Barbatia de acordo com as medidas morfométricas da concha consideradas no estudo. Legendda: NS- não significante; Asteriscos- significantes, onde: $\left({ }^{*}\right)$ baixa relevância, $\left({ }^{* *}\right)$ moderada relevância e $\left({ }^{* * *}\right)$ alta relevância.

\begin{tabular}{|c|c|c|c|}
\hline $\begin{array}{l}\text { Medida } \\
\text { (variável) }\end{array}$ & $\begin{array}{l}\text { Teste } \\
\text { Paramétrico }\end{array}$ & Comparação & $P$ value \\
\hline $\begin{array}{c}\mathbf{A C} \\
\text { (Posição do umbo) }\end{array}$ & Paramétrico & Barbatia candida $\times$ Barbatia domingensis & $* * * \quad p<0.001$ \\
\hline $\begin{array}{c}\mathbf{A E} \\
\text { (Comp. charneira) }\end{array}$ & $\begin{array}{c}\text { Não } \\
\text { Paramétrico }\end{array}$ & Barbatia candida $x$ Barbatia domingensis & $\star * * * \quad p<0.001$ \\
\hline $\begin{array}{c}\text { Al } \\
\text { (Altura da porção } \\
\text { anterior da } \\
\text { concha) }\end{array}$ & $\begin{array}{c}\text { Não } \\
\text { Paramétrico }\end{array}$ & Barbatia candida $x$ Barbatia domingensis & ${ }^{* \star *} \quad p<0.001$ \\
\hline $\begin{array}{c}\text { CB } \\
\text { (Altura do umbo) }\end{array}$ & $\begin{array}{c}\text { Não } \\
\text { Paramétrico } \\
\end{array}$ & Barbatia candida $x$ Barbatia domingensis & ${ }^{*} p<0.05$ \\
\hline $\begin{array}{c}\text { DJ } \\
\text { (Altura média da } \\
\text { concha) }\end{array}$ & $\begin{array}{c}\text { Não } \\
\text { Paramétrico }\end{array}$ & Barbatia candida $x$ Barbatia domingensis & $\star \star \star * \quad p<0.001$ \\
\hline $\begin{array}{c}\text { EK } \\
\text { (Altura da porção } \\
\text { posterior da } \\
\text { concha) }\end{array}$ & $\begin{array}{c}\text { Não } \\
\text { Paramétrico }\end{array}$ & Barbatia candida $x$ Barbatia domingensis & $* \star * * 0.001$ \\
\hline $\begin{array}{c}\text { LM } \\
\text { (Comp. da } \\
\text { concha) }\end{array}$ & $\begin{array}{c}\text { Não } \\
\text { Paramétrico }\end{array}$ & Barbatia candida $x$ Barbatia domingensis & $* * * \quad p<0.001$ \\
\hline $\begin{array}{c}\text { NO } \\
\text { (Comp. da porção } \\
\text { posterior da } \\
\text { concha) }\end{array}$ & Paramétrico & Barbatia candida $x$ Barbatia domingensis & 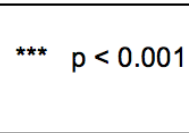 \\
\hline
\end{tabular}


Rocha, V. P. \& Matthews-Cascon, H. (2014). MORFOMETRIA DE ARCÍDEOS DO NORTE-NORDESTE DO BRASIL

Tabela V. Análises de variância entre os subgêneros de Anadara (Cunearca e Larkiria) e o gênero Lunarca de acordo com as medidas morfométricas da concha consideradas no estudo. Legendda: NS- não significante; Asteriscos- significantes, onde: $\left(^{*}\right)$ baixa relevância, $\left({ }^{* *}\right)$ moderada relevância e $\left({ }^{* * *}\right)$ alta relevância.

\begin{tabular}{|c|c|c|c|}
\hline $\begin{array}{l}\text { Medida } \\
\text { (variável) }\end{array}$ & $\begin{array}{l}\text { Teste } \\
\text { Paramétrico }\end{array}$ & Comparação & $P$ value \\
\hline $\mathbf{A C}$ & & Cunearca $x$ Larkiria & NS $p>0.05$ \\
\hline \multirow{2}{*}{$\begin{array}{l}\text { (Posição do } \\
\text { umbo) }\end{array}$} & Paramétrico & Cunearca x Lunarca & ${ }^{* * *} p<0.001$ \\
\hline & & Larkiria x Lunarca & $* * * \quad p<0.001$ \\
\hline $\mathrm{AE}$ & & Cunearca $x$ Larkiria & *** $\quad p<0.001$ \\
\hline (Comp. charneira) & Paramétrico & $\begin{array}{c}\text { Cunearca } x \text { Lunarca } \\
\text { Larkiria } \times \text { Lunarca }\end{array}$ & $\begin{array}{l}\text { NS } p>0.05 \\
* \star \star * \\
p<0.001\end{array}$ \\
\hline Al & & Cunearca $x$ Larkiria & NS $p>0.05$ \\
\hline (Altura da porção & Paramétrico & Cunearca $x$ Lunarca & $* * * \quad p<0.001$ \\
\hline $\begin{array}{c}\text { anterior da } \\
\text { concha) }\end{array}$ & & Larkiria x Lunarca & *** $p<0.001$ \\
\hline CB & & Cunearca $x$ Larkiria & $\begin{array}{ll}* \star * & p<0.001\end{array}$ \\
\hline \multirow[t]{2}{*}{ (Altura do umbo) } & Não & Cunearca $x$ Lunarca & $* \star * \quad p<0.001$ \\
\hline & Paramétrico & Larkiria x Lunarca & $\star \star \star * \quad p<0.001$ \\
\hline DJ & & Cunearca $x$ Larkiria & $\star \star \star * * \quad p<0.001$ \\
\hline (Altura média da & Paramétrico & Cunearca $x$ Lunarca & $* \star * \quad p<0.001$ \\
\hline concha) & & Larkiria x Lunarca & $\star \star \star * \quad p<0.001$ \\
\hline EK & & Cunearca $x$ Larkiria & *** $\quad p<0.001$ \\
\hline (Altura da porção & Não & Cunearca $x$ Lunarca & $* * \quad p<0.01$ \\
\hline $\begin{array}{l}\text { posterior da } \\
\text { concha) }\end{array}$ & Paramétrico & Larkiria x Lunarca & *** $\quad p<0.001$ \\
\hline LM & & Cunearca $x$ Larkiria & *** $\quad p<0.001$ \\
\hline \multirow{2}{*}{$\begin{array}{l}\text { (Comp. da } \\
\text { concha) }\end{array}$} & Não & Cunearca $x$ Lunarca & NS $p>0.05$ \\
\hline & Paramétrico & Larkiria x Lunarca & $* \star * * \quad p<0.001$ \\
\hline NO & & Cunearca $x$ Larkiria & NS $p>0.05$ \\
\hline (Comp. da porção & Paramétrico & Cunearca x Lunarca & $* * * \quad p<0.001$ \\
\hline $\begin{array}{l}\text { posterior da } \\
\text { concha) }\end{array}$ & & Larkiria x Lunarca & $* \star * * 0.001$ \\
\hline
\end{tabular}

A partir da análise dos dendrogramas das regiões da espécie Anadara notabilis, foi observado que as conchas em geral do Ceará e do Pará são mais similares. O mesmo ocorre para os espécimes de Lunarca ovalis. As conchas de Arca imbricata do Ceará são mais similares as do Rio Grande do Norte, assim como as de $A$. zebra do Ceará foram mais similares com as do Maranhão. Para os espécimes de Barbatia domingensis, as conchas do Amapá apresentaram-se mais similares com as do Pará (Figura 4).

\section{DISCUSSÃO}

As espécies de acrídeos analisadas nesse trabalho já possuíam registro para os estados brasileiros estudados (Rios, 2009). O tamanho médio das conchas dos arcídeos abordados apresentou-se consistente com as médias apresentadas na literatura (Diaz \& Puyana, 1994; Rios, 1994, 2009).

Nesse estudo, as medidas das variáveis morfológicas utilizadas foram relevantes em sua maioria para incrementar os dados informativos das espécies analisadas. Para a discriminação dos grupos internos de Arcidae foi possível identificar diferenças morfométricas nas conchas até nível de espécie. Ressalta-se que o trabalho não julga tais medidas como justificativa para separação dos mesmos, como se pode observar na Figura 2 - onde o dendograma de similaridade apresenta-se bastante heterogêneo, unindo espécies de gêneros distintos - mas como ferramenta auxiliar na identificação das mesmas. Nesse trabalho assume-se que as medidas foram úteis para observar a morfovariação das espécies de arcídeos presentes na costa Norte-Nordeste do Brasil.

Marko \& Jackson (2001), em estudo morfométrico anterior com espécimes da família Arcidae já haviam demonstrado a eficiência deste tipo análise. Utilizando-se de 14 medidas de variáveis morfológicas, os autores caracterizaram e diferenciaram pares de espécies, fósseis e do Recente, de três gêneros (Arca, Barbatia e Arcopsis). Ainda em Bivalvia, a análise da espécie Ledella pustulosa (Protobranchia), por exemplo, utilizando morfometria geométrica, foi relevante para explorar padrões de variação na forma da concha, desenvolver critérios morfométricos para distinção de subespécies e examinar padrões de distribuição das mesmas (Fuiman et al., 1999). 

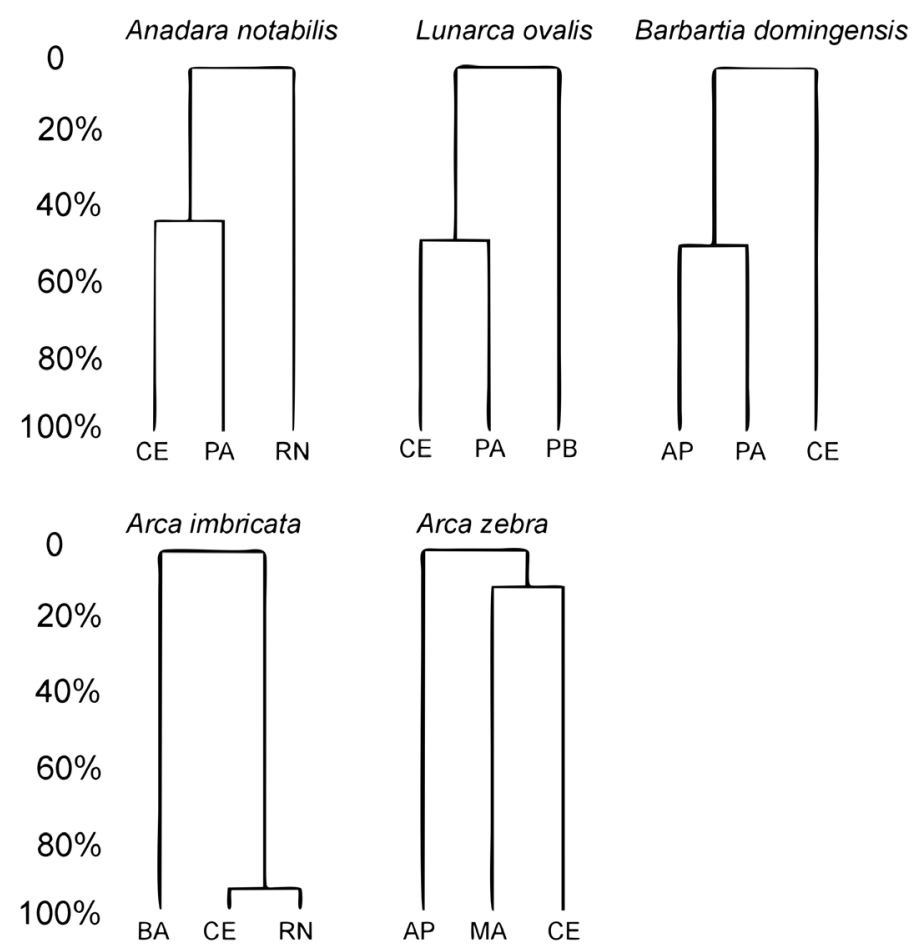

Figura 4. Dendograma de similaridade por distância Euclidiana das oito variáveis morfológicas medidas em arcídeos da costa NorteNordeste brasileira, mostrando uma possível relação das espécies de acordo com os estados do Brasil.

Estudos sobre populações e complexo de espécies com insetos comumente utilizam a morfometria geométrica, associada ou não a outra ferramenta, para compreensão desses grupos (Aytekin et al., 2007; Baylac et al., 2003; Hoffmann \& Shirriffs, 2002; Petrorius, 2005). Nestes trabalhos, as variações morfológicas observadas também são significativas.

Desenvolvimento ontogenético, adaptação a fatores geográficos locais, diversificação evolucionária de longo prazo são exemplos de processos biológicos que causam diferenças na forma entre espécies ou entre partes dos organismos (Zelditch et al., 2004). Em bivalves, a grande diversidade de hábitos de vida se reflete em uma ampla gama de variação morfológica, tanto na concha como na anatomia (Harper et al., 2000). Inclusive, problemas taxonômicos em bivalves se devem a fatores como falha na revisão de sinonímias, descrições simples das conchas, o não conhecimento mais aprofundado da espécie, bem como suas variações adaptativas (Bouchet et al., 2002; Bieler et al., 2013). Assim, as diferenças morfométricas podem estar relacionadas ao local onde os espécimes foram coletados. Trabalho como este são importantes, pois chama a atenção para as possíveis variações morfológicas entre espécimes.

Características como a prodisoconcha, já foram anteriormente relatadas, e corroboradas nesse estudo, como inúteis na identificação das espécies (Malchus, 2004a,b). O tamanho da prodisoconcha é uma característica que pode ter certa preferência a determinadas faixas de tamanho em alguns táxons superiores de bivalves. Por não se ter informações ontogenéticas claras, tal característica é utilizada apenas como caractere informativo e não como filogenético (Malchus, 2004b).

Os espécimes analisados nesse estudo que possuíam proximidade geográfica apresentaram similaridade morfológica. Sabendo que a costa brasileira possui ambientes diversos desenvolvidos no Quaternário, com regiões traçadas de acordo com parâmetros geomorfológicos, climáticos e oceanográficos (Silveira, 1968; Dominguez, 2006), podemos relacionar tais similaridades as características encontradas nestes ambientes.

Segundo Stanley (1970), salinidade e temperatura são os fatores mais limitantes na distribuição de moluscos bivalves. Assim, as conchas analisadas do litoral amazônico, que compreende desde o extremo norte do estado do Amapá e parte do Maranhão (porção oriental), poderiam apresentar menor similaridade com relação aos demais estados nordestinos devido à diferença de salinidade - influenciada pela desembocadura do rio Amazonas. Entretanto, conchas de Lunarca ovalis do Nordeste nesse estudo apresentaram-se mais similares com região Norte do que com conchas de outros estados nordestinos. 
Este fato pode ser explicado, pois a espécie também é encontrada em estuários, onde a salinidade também é reduzida (Diaz \& Puyana, 1994). A outra espécie a apresentar disparidade foi Anadara notabilis, que pode ser encontrada tanto em substratos arenosos (típicos do Nordeste), como associados a fanerógamas marinhas (Diaz \& Puyana, 1994; Rios, 2009). Assim os espécimes podem compartilhar situações ambientais semelhantes em regiões diferentes da costa brasileira.

Estudos morfológicos são bastante importantes para caracterização e entendimento dos grupos de bivalves. O presente estudo, com enfoque morfométrico, acrescentou novas informações conquiológicas para as espécies brasileiras, verificando as similaridades de espécimes da família para estados da costa Norte-Nordeste.

\section{AGRADECIMENTOS}

À Fundação Cearense de Apoio ao Desenvolvimento Científico e Tecnológico (FUNCAP) pela concessão da bolsa de mestrado para V. P. Rocha.

\section{REFERÊNCIAS BIBLIOGRÁFICAS}

Abbott, R.T. 1965. American Seashells. $7^{\text {a }}$ ed. D. Van Nostrand Company, Princeton, New Jersey, Toronto, Nova York, Londres, 348p.

Abbott, R.T. \& Dance, S.P. 1983. Compendium of Seashells - A color guide to more than 4.200 of the World's Marine Shells. $2^{\text {a }}$ ed. E.P. Dutton, Inc., Nova York, 411p.

Amaral, A.C.Z.; Rizzo, A.E. \& Arruda, E.P. 2006. Manual de identificação dos invertebrados da região Suldeste-Sul do Brasil. Editora EDUSP, São Paulo, 288p.

Aytekin, A.M.; Terzo, M.; Rasmont, P. \& Çagatay, N. 2007. Landmark based geometric morphometric analysis of wing shape in Sibiricobombus Vogt (Hymenoptera: Apidae: Bombus Latreille). Annales de la Société Entomologique de France (n.s.) 43(1): 95-102.

Baylac, M.; Villemant, C. \& Simbolotti, G. 2003. Combining geometric morphometrics with pattern recognition for the investigation of species complexes. Biological Journal of the Linnean Society 80: 89-93.

Bieler, R.; Mikkelsen, P. \& Giribet, G. 2013. Bivalvia - a discussion of known unknowns. American Malacological Bulletin 31(11): 123-133.

Broom, M. J. 1983. Gonad Development and Spawning Anadara granosa (L.) (Bivalvia: Arcidae). Aquaculture 30: 211-219.
Bouchet, P.; Lozouet, P.; Maestrati, P. \& Heros, V. 2002. Assessing the magnitude of species richness in tropical marine environments: exceptionally high numbers of molluscs at a New Caledonia site. Biological Journal of Linnean Society 75: 421-436.

Diaz, J.M.M. \& Puyana, M.H. 1994. Mosluscos del caribe Colombiano. Colciencias Y Fundación Natura, Santafé de Bogotá, 291 p.

Dominguez, J.M.L. 2006. The Coastal Zone of Brazil: an overview. Journal of Coastal Research 39: 16-20.

Francisco, J.A.; Tenório, D.O.; Barros, J.C.N.; Silva, J.G.A. \& Silva, G.F. 2011. Shell morphometry of three species of the genus Nuculuna Link, 1807 (Bivalvia, Protobranchia, Nuculanidae) from continental shelf and slope Northeastern Brazil. Tropical Oceanography 39(1): 22-26.

Francisco, J.A.; Barros, J.C.N \& Lima, S.F.P. 2012. Five new species of Arcidae from Brazil with description of new genus: Paranadara (Mollusca, Bivalvia). Journal of the Marine Biological Association of the United Kingdom 92(5): 1139-1150.

Fuiman, L.A; Gage, J.D. \& Lamont, P.A. 1999. Shell morphometry of the deep sea protobranch bivalve Ledella pustulosa in the Rockall Trough, north-east Atlantic. J. Mar. Biol. Assoc. UK. 79: 661-671.

Harper, E.M.; Taylor, J.D. \& Crame, J.A. 2000. Unravelling the evolutionary biology of the Bivalvia: a multidisciplinary approach. Geol. Soc. Spec. Publ. 177:1-19.

Hoffmann, A.A. \& Shirriffs, J. 2002. Geographic variation for wing shapein Drosophila serrata. Evolution 56(5): 1068-1073.

Huber, M. 2010. Compendium of bivalves. A full-color guide to 3'300 of the world's marine bivalves. A status on Bivalvia after 250 years of research. ConchBooks, 1 CD-Rom, 901p.

La Perna, R. \& D'Abramo, M. 2009. Morphometric and systematic study on three Acanthocardia species from the Mediterranean Pleistocene (Mollusca, Bivalvia, Cardiidae). Geodiversitas 31(3): 669-682.

Malchus, L. 2004a. Constraints in the ligament ontongeny and evolution of Pteriomorphian Bivalvia. Paleontology 47(6): 1539-1574.

Malchus, L. 2004b. Early ontogeny of Jurassic bakevelliids and their bearing on bivalve evolution. Acta Palaeontol. Pol. 49(1): 85-110.

Marko, P.B. \& Jackson, J.B.C. 2001. Patterns of Morphological Diversity Among and Within Arcid Bivalve Species Pairs Separated by the Isthmus of Panama. J. Paleontol. 75(3): 590-606.

Monteiro, L.R. \& Reis, S.F. 1999. Princípios de Morfometria Geométrica. Holos Editora, Ribeirão Preto, 188p. 
Nevesskaja, L.A. 2008. Dynamics of taxonomic diversity of bivalves in the Phanerozoic. Paleontological Journal 42(4): 3-11.

Nevesskaja, L. A. 2009. Principles of systematics and the system of bivalves. Paleontological Journal 43(1): 3-13.

Oliver, P.G. \& Holmes, A.M. 2006. The Arcoidea (Mollusca: Bivalvia): a review of the current phonetic-based systematics. Zoological Journal of the Linnean Society 148: 237-251.

Passos, F.D. \& Birman, A. 2009. New records of Bentharca asperula (Dall, 1881) (Mollusca, Bivalvia, Arcidae) from Brazil. Biota Neotropica 9(4): 281-283.

Peharda, M.; Mladineo, I; Bolotin, J.; Kekez, L. \& Skaramuca, B. 2006. The reproductive cycle and potencial protandric development of the Noah's Ark shell, Arca noae L.: Implications for aquaculture. Aquaculture 252: 317-327.

Petrorius, E. 2005. Using geometric morphometrics to investigate wing dimorphism in males and females of Hymenoptera - a case study based on the genus Tachysphex Kohl (Hymenoptera: Sphecidae: Larrinae). Australian Journal of Entomology 44: 113-121.

Purchon, R.D. \& Brown, D. 1969. Phylogenetic interrelationships among families of bivalve moluscs. Malacologia 9(1): 163-171.

Rios, E. C. 1994. Seashells of Brazil. Editora da Fundação Universidade de Rio Grande, Rio Grande, 368p.

Rios, E.C. 2009. Compendium of Brazilian Sea Shells. Evangraf, Rio Grande, 668p.Rohlf, F.J. 2000. Geometric morphometrics and phylogeny. Graduate Studies in Ecology and Evolution, Nova York, 15p.

Submetido: Maio/2014

Revisado: Junho/2015

Aceito: Setembro/2015
Roopnarine, P.D.; Signorelli, J. \& Laumer, C. 2008. Systematic, Biogeographic, and Microhabitatbased morphometric variation of the bivalve Anomalocardia squamosa (Bivalvia: Veneridae: Chioninae) in Thailand. The Raffles Bulletin of Zoology 18: 90-98.

Silina, A. 2006. Spatial heterogeneity and long-term changes in bivalve Anadara broughtoni population: influence of river run-off and fishery. Ocean Science Journal 41 (4): 211-219.

Silveira, J.D. 1968. Morfologia do litoral. In: Azevedo, A. Brasil: A terra e o homem. Vol.1: As bases físicas, $2^{\mathrm{a}}$ ed. Companhia Editora Nacional, São Paulo, p. 253-305.

Simone, L.R.L. 2009. A new species of Acar (Bivalvia, Arcidae) from São Pedro e São Paulo Archipelago, Brazil. Novapex 10(1): 9-16.

Simone, L.R.L. \& Chichvarkhin, A. 2004. Comparative Morphological Study of Four Species of Barbatia Occurring on the Southern Florida Coast (Arcoidea, Arcidae). Malacologia 46(2): 355-379.

Stanley, S.M. 1970. Relation of shell from to life habits in the Bivalvia (Mollusca). Memoirs of the Geological Society of America 125: 1-296.

Warmke, G.S. \& Abbott, R.T. 1962. Caribbean Seashells - A guide to the Marine Mollusks of Puerto Rico and the West Indian Islands, Bermuda and the Lower Florida Keys. $4^{a}$ ed. Livingston Company, Wynnewood, Pensylvania, 541p.

Zelditch, M.L.; Swiderski, D.L.; Sheets, H.D. \& Fink, W.L. 2004. Geometric Morphometrics for Biologists: A primer. Elsevier Academic Press, Nova York e Londres, 437p. 